\title{
Curva de aprendizado em cirurgia aórtica videolaparoscópica: estudo experimental em porcos
}

\author{
Laparoscopic aortic surgery learning curve: \\ experimental study in pigs
}

\section{Ricardo de Alvarenga Yoshida ${ }^{1}$, Winston Bonetti Yoshida ${ }^{2}$, Hamilton de Almeida Rollo ${ }^{2}$, Ralf Kolvenbach ${ }^{3}$, Sílvia Elaine Rodolfo de Sá Lorena ${ }^{4}$}

\section{Resumo}

Contexto: A cirurgia videolaparoscópica (CVL) vem evoluindo como alternativa cirúrgica menos invasiva para o tratamento da doença aterosclerótica oclusiva aorto-ilíaca e do aneurisma da aorta abdominal. Poucos estudos avaliaram objetivamente a curva de aprendizado com essa técnica em cirurgia vascular.

Objetivo: Avaliar objetivamente os tempos e a evolução de cada passo cirúrgico e demonstrar a exeqüibilidade dessa técnica.

Métodos: Entre outubro 2007 e janeiro de 2008, dois cirurgiões vasculares iniciantes na CVL operaram, após cursos e treinamentos, seis porcos consecutivos, com dissecção aórtica e interposição de um enxerto de dácron em um segmento da aorta infra-renal abdominal, com técnica totalmente laparoscópica.

Resultados: Todos os tempos cirúrgicos foram decrescentes ao longo do estudo, apresentando redução de $45,9 \%$ no tempo total de cirurgia, $85,8 \%$ no tempo de dissecção da aorta, $81,2 \%$ na exposição da aorta, $55,1 \%$ no clampeamento total, $71 \%$ na confecção da anastomose proximal e 64,9\% na anastomose distal.

Conclusão: $\mathrm{O}$ presente estudo mostrou que os resultados técnicos satisfatórios da CVL vascular ocorreram somente após longa curva de aprendizado, que foi decrescente ao longo do tempo, à medida que aumentou a experiência e vivência com os materiais e com a visão não-estereoscópica. Essa técnica pode ser realizada com perfeição por cirurgiões vasculares desde que façam cursos especializados, com treinamento em simuladores e animais, e desde que busquem constante aprimoramento a fim de conseguir resultados similares aos obtidos com a cirurgia convencional.

Palavras-chave: Cirurgia videoassistida, aorta, doenças vasculares, aprendizagem, modelos animais.

\section{Abstract}

Background: Laparoscopic aortic surgery (LAS) is evolving as a minimal invasive alternative technique for the treatment of abdominal aortic aneurysms and peripheral aortoiliac arterial disease. Few articles have objectively evaluated the technique learning curve in vascular surgery.

Objective: The aim of the present experimental study was to demonstrate surgical feasibility and evaluate each surgical step of LAS.

Methods: Between October 2007 and January 2008, two beginning surgeons attended laparoscopic courses and operated on six consecutive pigs with a total laparoscopic retroperitoneal aortic dissection and interposition of a Dacron tube graft in the infrarenal aortic segment. Both anastomoses were performed under total laparoscopic technique.

Results: All surgical time curves decreased along time: $45.9 \%$ in total surgical time, $85.8 \%$ in dissection time, $81.2 \%$ in aortic exposure, $55.1 \%$ in total cross-clamping, $71 \%$ in proximal anastomosis and $64.9 \%$ in distal anastomosis.

Conclusion: This experimental study showed that satisfactory results with LAS were only reached after a long learning curve. However, this learning curve decreased along time, as experience with the technique, materials and non-stereoscopic vision improved. Vascular surgeons should attend practical courses and should have training opportunities in simulators and animals to achieve results similar to those of conventional surgery.

Keywords: Video-assisted surgery, aorta, vascular diseases, learning, animal models.

1. Pós-graduando. Colaborador, Disciplina de Angiologia e Cirurgia Vascular, Faculdade de Medicina de Botucatu, Universidade Estadual Paulista (UNESP), Botucatu, SP.

2. Livre-docente. Professor adjunto, Faculdade de Medicina de Botucatu, UNESP, Botucatu, SP.

3. Professor titular. Chefe, Serviço de Cirurgia Vascular e Endovascular, Hospital Augusta, Dusseldorf, Alemanha.

4. Doutoranda, Departamento de Cirurgia e Anestesiologia Veterinária, Faculdade de Medicina Veterinária e Zootecnia (FMVZ), UNESP, Botucatu, SP.

Conflitos de interesse: Johnson \& Johnson, Karl Storz Alemanha e Brasil, H. Strattner e Astus disponibilizaram equipamentos e materiais, e H. Strattner patrocinou o curso de CVL.

Artigo submetido em 11.06.08, aceito em 20.08.08.

J Vasc Bras. 2008;7(3):231-238.

Copyright@ 2008 by Sociedade Brasileira de Angiologia e de Cirurgia Vascular 


\section{Introdução}

A cirurgia videolaparoscópica (CVL) vem evoluindo como alternativa cirúrgica menos invasiva para o tratamento de diversas doenças ${ }^{1-6}$. Inicialmente, o mesmo interesse e entusiasmo pela CVL não foi observado na cirurgia vascular ${ }^{1,7}$. Dificuldades técnicas iniciais na dissecção e clampeamento vascular, falta de materiais e acessórios específicos, dificuldade técnica e grande curva de aprendizado não estimularam muitos cirurgiões vasculares a se dedicarem à técnica videolaparoscópica $^{1,7,8}$.

No entanto, alguns centros, principalmente na Europa e Canadá, alcançaram um avanço notável com a CVL, tornando essa técnica operatória vascular acessível e padronizada ${ }^{1,3,9-12}$.

As vantagens da CVL em cirurgia vascular para reconstruções aórticas são as mesmas de outras CVL gastrintestinais ou urológicas, ou seja, são menos invasivas, apresentam trauma cirúrgico reduzido, menor incisão abdominal e dor pós-operatória, alimentação oral precoce e permanência hospitalar reduzida ${ }^{5,8,13-17}$. As cirurgias aórticas laparoscópicas tiveram resultados similares ou melhores do que os obtidos com a cirurgia aberta convencional quando comparados os parâmetros hemodinâmicos, metabólicos e inflamatórios ${ }^{17-21} \mathrm{e}$ o seguimento em longo prazo ${ }^{1,3,22}$.

Uma das limitações da CVL vascular está na longa curva de aprendizado necessária para a obtenção do sucesso técnico ${ }^{1,3}$. Poucos estudos foram feitos no sentido de avaliar prospectivamente e especificamente esse pormenor da técnica ${ }^{23-28}$.

Assim sendo, este estudo experimental pretende avaliar objetivamente os tempos e a evolução de cada passo cirúrgico e demonstrar a exeqüibilidade da técnica.

\section{Métodos}

Este estudo foi realizado no período de março de 2007 a janeiro de 2008 como parte de projeto de pósgraduação, tendo sido aprovado pelo Comitê de Ética em Pesquisa Animal. Foi feita análise prospectiva dos tempos necessários para se completar cada etapa da técnica operatória em porcos. Foi feita apenas análise estatística descritiva dos dados.
Durante este estudo piloto em porcos, os autores realizaram cursos de videocirurgia geral ( 8 e 30 horas) e videocirurgia ginecológica (32 horas). Fizeram também treinamento intensivo de suturas e nós em caixa preta (cerca de 60 horas).

A seguir, seis porcos Landrace, de ambos os sexos, com peso variando entre 20 e $30 \mathrm{~kg}$, em boas condições de saúde, foram submetidos a cirurgia totalmente laparoscópica. Todas as cirurgias foram realizadas no Laboratório de Cirurgia Experimental do Departamento de Cirurgia e Ortopedia da Faculdade de Medicina de Botucatu, na Universidade Estadual Paulista (UNESP) (Figura 1A).

Os animais foram submetidos a jejum alimentar de 14 horas, e o acesso à água foi livre. Foi ministrada medicação pré-anestésica com associação de acepromazina $(0,1 \mathrm{mg} / \mathrm{kg})$, xilazina $(1 \mathrm{mg} / \mathrm{kg})$, cloridrato de cetamina $(8 \mathrm{mg} / \mathrm{kg})$ e morfina $(0,5 \mathrm{mg} / \mathrm{kg})$ pela via intramuscular. Quinze minutos após a aplicação, a veia central da orelha foi puncionada com agulha $22 \mathrm{G}\left(\right.$ Abbocath $\left.^{\circledR}\right)$, e a anestesia induzida com a administração de cloridrato de cetamina ( $2 \mathrm{mg} / \mathrm{kg}$ ) e de propofol (4 $\mathrm{mg} / \mathrm{kg}$ ) pela via intravenosa. A manutenção foi realizada com halotano. Em seguida, o animal foi posicionado em decúbito ventral na mesa de procedimentos, para entubação orotraqueal com sonda 7,0. Foi instituída ventilação controlada a volume, através do respirador modelo 674 do aparelho de anestesia K. Takaoka (mod. 2.600, Nikkei, Brasil). O volume corrente foi padronizado em $20 \mathrm{~mL} / \mathrm{kg}^{-1} \mathrm{de} \mathrm{O}_{2}$, e a freqüência respiratória, em 10 a 12 mov $\min ^{-1}$, para manter a pressão expiratória final de $\mathrm{CO}_{2}\left(\mathrm{PETCO}_{2}\right)$ entre 30 a $35 \mathrm{mmHg}$. A saturação de $\mathrm{O}_{2}$ foi medida por oximetria de pulso, com sensor na língua do animal, em valor superior a 97\%. A fluidoterapia foi realizada com infusão de $10 \mathrm{~mL} / \mathrm{kg}$ de Ringer lactato.

\section{Técnica cirúrgica}

Todas as cirurgias foram realizadas por um cirurgião principal e por um auxiliar, que acumulou as funções de câmera e auxiliar cirúrgico. Os equipamentos usados foram os conjuntos para CVL da Storz, da Astus ou da Olympus, conforme disponibilidade. 
Após o posicionamento do animal em decúbito lateral esquerdo (Figura 1B), o pneumoperitôneo foi iniciado após punção com agulha de Veress no espaço subcostal esquerdo e mantido com pressão de $12 \mathrm{~mm}$ $\mathrm{Hg}$ de $\mathrm{CO}_{2}$. A seguir, um trocar de 11 milímetros foi colocado acima do umbigo para introdução da ótica de $30^{\circ}$. Dois outros trocars de 11 milímetros (Endopath, Johnson \& Johnson) foram colocados na linha média, abaixo e acima do umbigo. Além disso, outros quatro trocars foram colocados em torno do lado esquerdo, tendo como referência o rebordo costal, a linha axilar média, a musculatura dorsal e a crista ilíaca (Figura 1B).

A exposição da aorta se iniciou com a mobilização medial do hemicólon esquerdo, rim esquerdo e flexura esplênica, usando grasper, tesouras laparoscópicas e bisturi harmônico (ULTRACISION ${ }^{\circledR}$, Johnson \& Johnson). A fáscia de Gerota foi exposta, e o hemicólon esquerdo deslocado medialmente, seguindo as orientações da técnica Apron ${ }^{29}$ (Figuras 2A e B). Após a completa dissecção da aorta e da sua bifurcação (Figuras $2 \mathrm{C}$ e 2D), as artérias lombares aórticas foram ligadas com Liga-clip ${ }^{\circledR}$ (Johnson \& Johnson) ou eletrocoaguladas com bisturi harmônico (ULTRACISION ${ }^{\circledR}$, Johnson \& Johnson). Um clampe aórtico laparoscópico (Clampe Vascular Laparoscópico Storz, ref. 49310VC) foi posicionado na aorta infra-renal, e outro clampe na aorta logo acima de sua bifurcação, através de portais localizados abaixo do rebordo costal e da crista ilíaca, respectivamente. Após administração de heparina endovenosa (100 UI/kg), a aorta foi clampeada e seccionada (Figura 2E). Uma aortotomia longitudinal de cerca de $6 \mathrm{~cm}$ foi feita entre os dois clampes (Figura 2F). A prótese vascular de dácron $6 \mathrm{~mm}$ foi introduzida através de um dos portais, e a anastomose proximal realizada laparoscopicamente, com sutura contínua com fios de Prolene ${ }^{\circledR}$ 4-0 (Johnson \& Johnson), usando porta-agulha laparoscópico (Storz, ref. 49310NL), seguindo a técnica de Coggia et al. ${ }^{30}$. Após o término da anastomose proximal, liberou-se temporariamente o fluxo sangüíneo para observar sangramentos remanescentes, os quais, quando presentes, foram corrigidos com pontos adicionais (Figura 3A). Após novo clampeamento, a anastomose distal foi realizada da mesma maneira (Figura 3B).

Terminadas as anastomoses, liberou-se o fluxo na anastomose distal, deixando sair o ar da prótese, e em

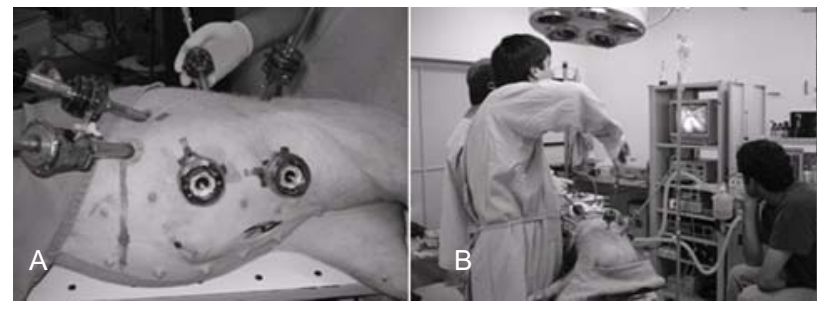

Figura 1 - A) Posição dos trocares; B) conjunto experimental no Laboratório de Cirurgia Experimental da Faculdade de Medicina de Botucatu, Universidade Estadual Paulista

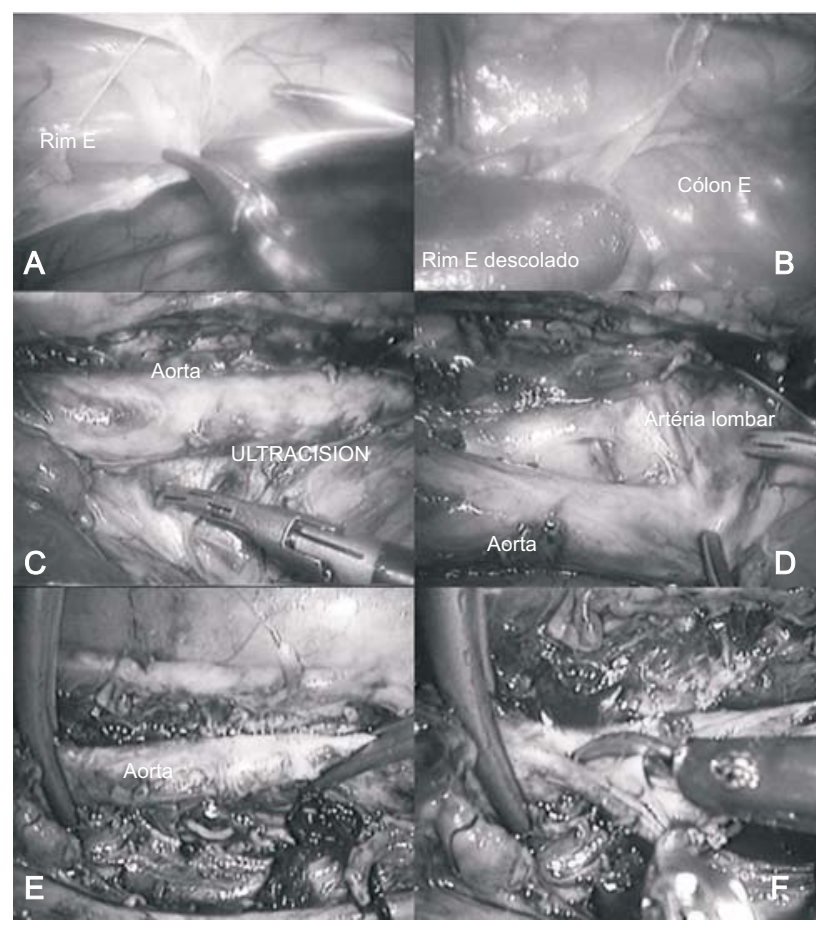

Figura 2 - A, B) Exposição retroperitoneal da aorta (Apron technique); C, D) dissecção da aorta; E) clampeamento total da aorta; F) aortotomia longitudinal de mais ou menos $6 \mathrm{~cm}$

seguida na anastomose proximal, restituindo assim a perfusão para os membros inferiores (Figura 3C). Os sangramentos remanescentes pelas anastomoses proximal e distal foram corrigidos com pontos adicionais. $\mathrm{O}$ enxerto foi recoberto pelo hemicólon esquerdo e pelo peritôneo, recolocando-se o animal em decúbito dorsal horizontal. 


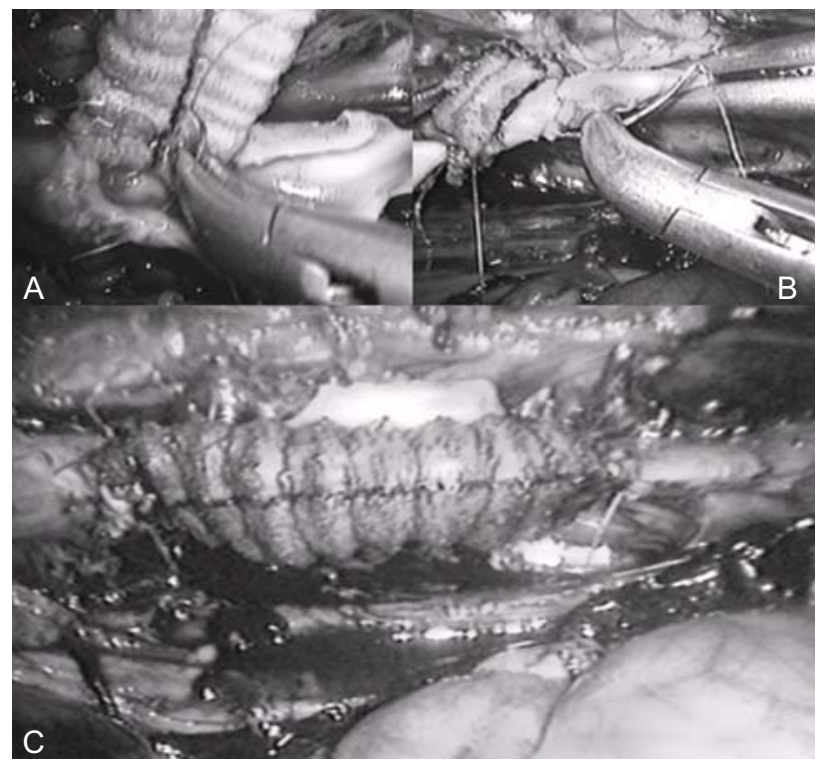

Figura 3 - Anastomoses realizadas com técnica totalmente laparoscópica: A) anastomose proximal; B) anastomose distal; C) aspecto final

Ao final do procedimento, realizou-se eutanásia do animal com cloreto de potássio endovenoso, sob anestesia geral.

\section{Resultados}

O tempo cirúrgico total médio, de clampeamento total e da anastomose distal só puderam ser calculados a partir da terceira cirurgia, pois inicialmente não foi possível completar todos os tempos. Problemas técnicos iniciais com a realização da cirurgia totalmente laparoscópica, em especial com a confecção da anastomose, demandaram um tempo cirúrgico muito elevado.

O tempo cirúrgico total médio foi de 185 minutos (130-240 min), apresentando tempos parciais decrescentes de 240, 180, 180 e 130 minutos para as cirurgias realizadas nos porcos 3, 4, 5 e 6, respectivamente (redução de $45 \%$ entre a terceira e a última cirurgia) (Figura 4). Os tempos médios de dissecção e exposição da aorta antes do clampeamento foram de 20 minutos (5-35 min), com tempos decrescentes de 35, 21, 14, 16, 10 e 5 minutos (redução de $85 \%$ entre a primeira e a última cirurgia), e de 28,5 minutos (9-48 min), com tempos decrescentes de 48, 14, 13, 19, 9 e 9 minutos (redução de $81,2 \%$ entre a primeira e a última cirurgia), respectivamente (Figura 5). O tempo médio de cirurgia para a

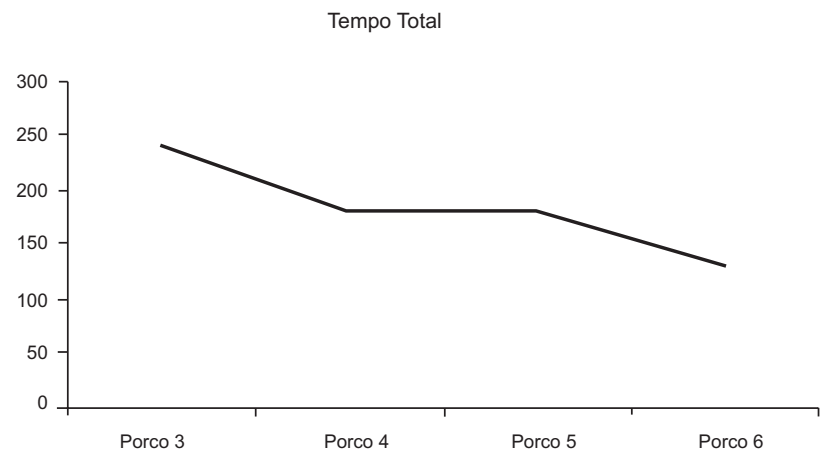

Figura 4 - Tempo cirúrgico total (min)

exposição retroperitoneal da aorta, antes do clampeamento cirúrgico, realizada com a técnica Apron, bem como sua dissecção e a ligadura externa das artérias lombares com clipes (Liga-clip ${ }^{\circledR}$, Johnson \& Johnson) ou eletrocoagulação com bisturi harmônico (ULTRACISION $^{\circledR}$, Johnson \& Johnson) foi de 48,5 minutos (decrescente de 83 a $14 \mathrm{~min}$ ). O tempo médio de clampeamento total foi de 115, 5 minutos, com tempos decrescentes de 157, 166, 82 e 74 minutos (redução de 52,8\% entre a terceira e a última cirurgia), para as cirurgias realizadas nos porcos 3, 4, 5 e 6, respectivamente (Figura 6).

O tempo médio da anastomose proximal foi de 64,5 minutos, com tempos decrescentes de 100, 50, 40, 38, 46 e 29 minutos (redução de $71 \%$ entre a primeira e a última cirurgia) (Figura 7). Em dois porcos, foi necessária a aplicação de pontos adicionais hemostáticos. Para a anastomose distal, o tempo médio foi de 47 minutos, com tempos decrescentes 57, 38, 48 e 37 minutos (redução de

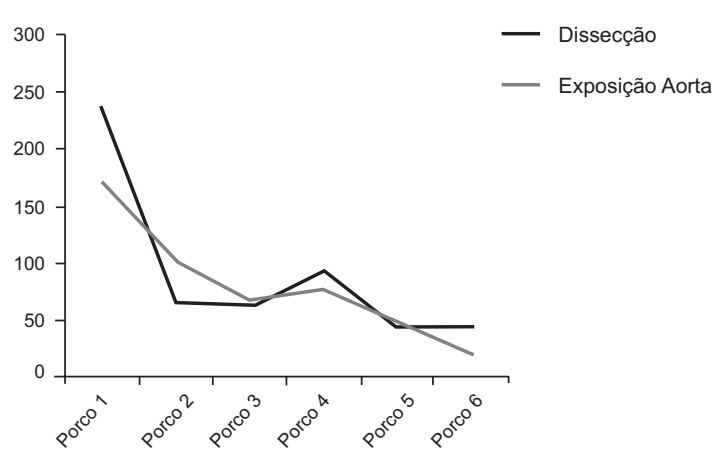

Figura 5 - Tempos de dissecção e exposição da aorta (min) 


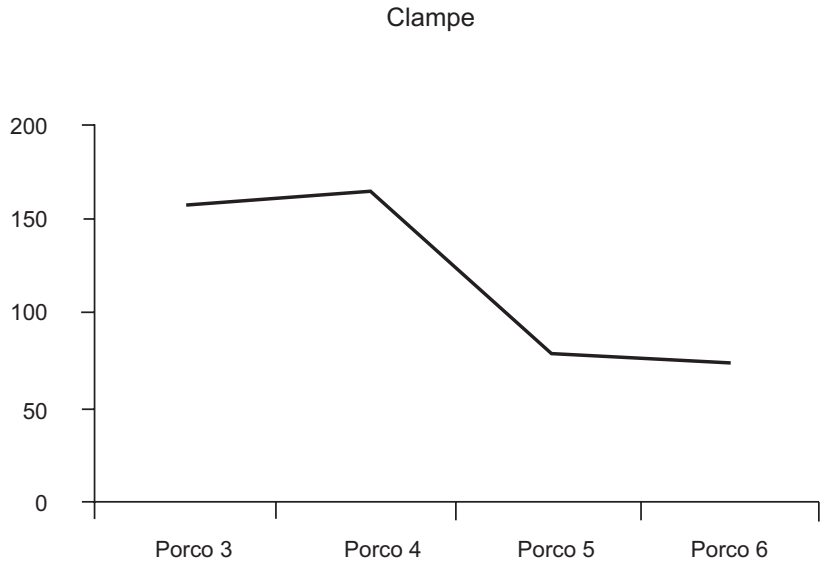

Figura 6 - Tempo de clampeamento (min)

$35 \%$ entre a terceira e a última cirurgia) para as cirurgias realizadas nos porcos $3,4,5$ e 6 , respectivamente (Figura 7).
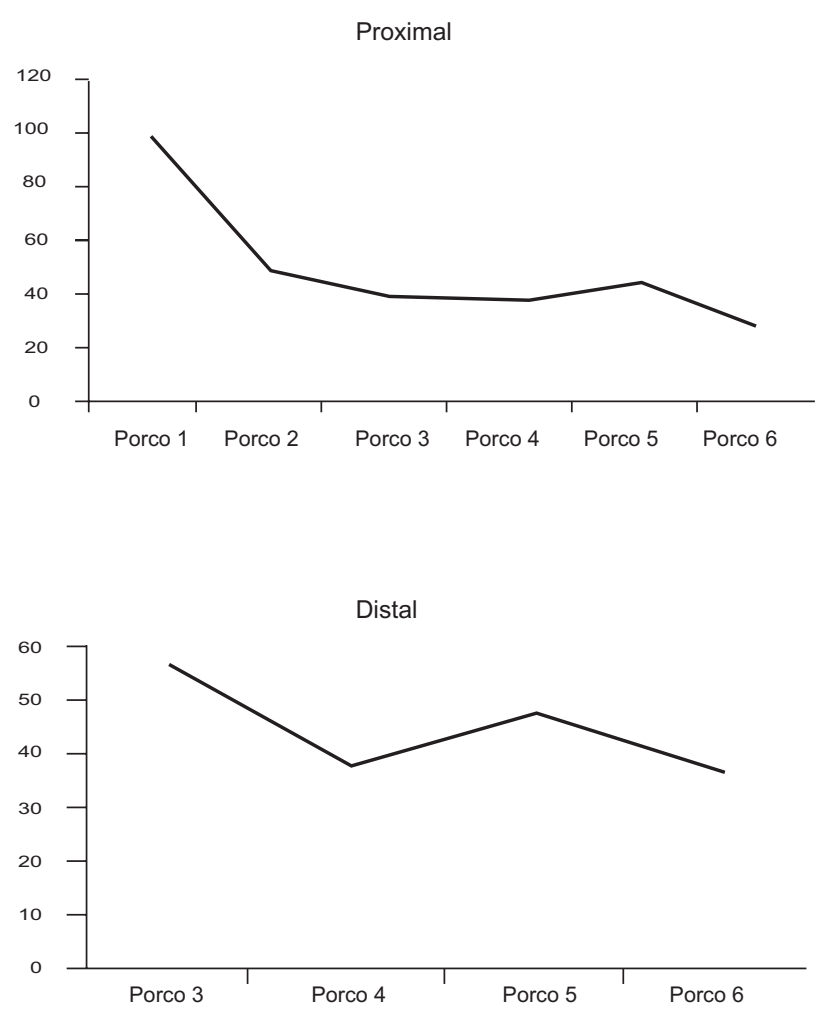

Figura 7 - Tempo da anastomose proximal e distal (min)
Em nenhum caso houve lesões inadvertidas de vasos sangüíneos ou de estruturas vizinhas. Na segunda cirurgia, um animal foi a óbito antes do sacrifício por problemas no aparelho de anestesia, relacionados à má difusão dos gases anestésicos.

Foi realizada autópsia na sexta cirurgia, para a verificação de ambas as anastomoses, não sendo evidenciados defeitos técnicos, estenoses ou vazamentos.

No inventário final da cavidade abdominal, não foram evidenciadas lesões de órgãos intracavitários em nenhuma das seis cirurgias.

\section{Discussão}

Descrita primeiramente por Dion em $1993^{31}$, a cirurgia aórtica videolaparoscópica evoluiu até ser empregada rotineiramente na maioria dos pacientes portadores de doença oclusiva aorto-ilíaca ou aneurisma da aorta abdominal (AAA) ${ }^{3,10,11,32-35}$, além de ser coadjuvante em procedimentos endovasculares, caracterizando técnicas híbridas ${ }^{15,33,36-38}$. Atualmente, três técnicas principais são empregadas para reconstruções da aorta: a CVL videoassistida (HALS ou hand-assisted laparoscopic surgery), a cirurgia laparoscópica minimamente invasiva (minilaparotomia) e a cirurgia totalmente laparoscópica ${ }^{1}$.

No Augusta Hospital (serviço do Prof. Kolvenbach, em Dusseldorf, Alemanha), no período de 2002 a 2006, foram operados 131 pacientes com diagnóstico de AAA, utilizando-se a técnica totalmente laparoscópica. Após um período médio de seguimento de 39 meses, 127 pacientes encontravam-se vivos e seguidos regularmente ${ }^{3}$.

No entanto, para se atingir tais resultados, foi necessário atravessar uma longa curva de aprendizado. No presente estudo, observamos que não foi possível terminar as primeiras cirurgias e que o tempo de execução da anastomose proximal foi, inicialmente, maior que o tempo total das últimas cirurgias completas. Porém, ao longo do tempo, todas as curvas temporais de tempo total, dissecção e exposição da aorta, anastomoses e clampeamento tiveram um perfil decrescente, em função não só da experiência adquirida com as cirurgias mas também de treinamentos em caixa preta e cursos teórico-práticos em CVL, realizados no período. Esses 
cursos municiaram os autores com recursos, técnicas de nós e suturas, além de protocolos e treinamentos em simuladores, os quais, embora voltados mais para cirurgia geral, ginecológica e urológica, contribuíram para a redução do tempo operatório. Outro fator importante para a diminuição desse tempo foi o emprego do bisturi harmônico, que facilitou a dissecção e exposição da aorta devido ao seu efetivo poder de corte e coagulação, sem dissipar calor ou provocar fumaça, minimizando sangramentos indesejáveis e podendo ser usado em tecidos periaórticos, que são freqüentemente sangrantes. No entanto, a diminuição dos tempos cirúrgicos poderia ser ainda mais significativa se as operações tivessem sido realizadas por um cirurgião principal e dois auxiliares, conforme sugerido pela literatura, pois o acúmulo das funções de câmera e auxiliar cirúrgico por um único cirurgião auxiliar dificulta a realização das anastomoses.

No Departamento de Cirurgia Vascular da Universidade de Pisa, Itália, chefiado pelo Prof. Mauro Ferrari, observou-se significativa redução nos tempos cirúrgicos e de clampeamento, após curva de aprendizado, em pelo menos 30 pacientes, corroborando os dados do presente estudo sobre os tempos operatórios decrescentes durante a curva de aprendizado à medida que vão se somando as experiências dos sucessivos $\operatorname{casos}^{1,11}$.

Dion et al. $^{23}$, em estudo experimental envolvendo também seis porcos, demonstraram a exeqüibilidade da sutura terminolateral realizada laparoscopicamente, apresentando tempo médio cirúrgico total de 198 minutos (170-240 min). Fusco et al. ${ }^{36}$ obtiveram tempo de clampeamento total para cirurgia aortofemoral de 1 hora em um porco operado. Em comparação com o presente estudo, os tempos médios foram semelhantes na realização das anastomoses $(64,5 \mathrm{~min}$ para anastomose proximal e 47 min para anastomose distal) e no tempo cirúrgico médio total (185 min). A diferença dos tempos médios na dissecção e exposição da aorta (48,5 min) antes do clampeamento e no clampeamento total (115, $5 \mathrm{~min}$ ) deveu-se aos diferentes procedimentos cirúrgicos realizados nesses estudos ${ }^{36}$. A cirurgia aórtica videolaparoscópica somente poderá ser executada quando o cirurgião e a equipe bem treinada, composta por pelo menos um auxiliar responsável pela câmera e outro auxiliar pela cirurgia propriamente dita, adquirirem habilidades laparoscópicas básicas ${ }^{1,3,10,17,33}$ e tiverem perfeito entrosamento. Não importa se essas habilidades vierem dos procedimentos cirúrgicos videolaparoscópicos de cirurgia geral ou de cursos de CVL voltados para outras especialidades $^{1,3}$. No Brasil não há, no momento, cursos voltados para cirurgia vascular ou serviços de cirurgia vascular que empreguem rotineiramente a técnica videolaparoscópica vascular. Além disso, não há cirurgias laparoscópicas vasculares consideradas "menores" para se iniciar e treinar a técnica, como ocorre em outras especialidades $^{1,3}$. Os interessados deverão buscar conhecimento nos cursos gerais de laparoscopia e treinamento em simuladores e animais de experimentação antes de se aventurarem no tratamento de pacientes vasculares com a CVL. Faz-se necessário o emprego de materiais de boa qualidade e específicos para laparoscopia vascular.

Considera-se como limitação para o desenvolvimento dessa técnica a prolongada curva de aprendizado, sendo atualmente difícil formar uma equipe bem treinada, devido a todos os aspectos apontados anteriormente $^{1}$. Além disso, os equipamentos, materiais e clampes não são baratos. No entanto, a CVL aórtica, que nada mais é do que a cirurgia convencional realizada por via laparoscópica, vem apresentando bons resultados associados à elegância técnica, baixa invasividade e segurança; tais resultados devem ser a motivação para que os cirurgiões vasculares ingressem nesse aprendizado $^{1,3}$.

O presente estudo mostrou que os resultados técnicos satisfatórios da CVL vascular ocorrem somente após uma longa curva de aprendizado, que é decrescente à medida que aumenta a experiência e a vivência com os materiais e com a visão não-estereoscópica. Essa técnica pode ser realizada com sucesso por cirurgiões vasculares, da mesma forma que os cirurgiões gerais, ginecologistas e urologistas realizam cirurgias laparoscópicas complexas. O treinamento intensivo, os cursos de laparoscopia e a busca constante de aprimoramento técnico devem ser perseguidos para se obter os mesmos resultados alcançados na cirurgia convencional. 


\section{Agradecimentos}

Os autores agradecem às empresas Johnson \& Johnson - Divisão Ethicon Endo-Surgery, Karl Storz Alemanha e Brasil, H. Strattner e Astus, pelo apoio e disponibilização de equipamentos e materiais para esse projeto. Agradecem também a H. Strattner pelo patrocínio do curso de CVL.

\section{Referências}

1. Kolvenbach R, Yoshida RAY. Cirurgia aórtica videolaparoscópica. In: Maffei FHA, ed. Doenças vasculares periféricas. $4^{\mathrm{a}}$ ed. Rio de Janeiro: Guanabara Koogan; 2008. v. 2. "No prelo".

2. Lin JC, Kolvenbach R, Schwierz E, Wassiljew S. Total laparoscopic aortofemoral bypass as a routine procedure for the treatment of aortoiliac occlusive disease. Vascular. 2005;13:80-3.

3. Kolvenbach R. Total laparoscopic aortic aneurysm surgery. Acta Chir Belg. 2006;106:36-9.

4. Coggia M, Javerliat I, Di Centa I, et al. Total laparoscopic infrarenal aortic aneurysm repair: preliminary results. J Vasc Surg. 2004;40:448-54.

5. Coggia M, Di Centa I, Javerliat I, Alfonsi P, Kitzis M, GoeauBrissonniere OA. Total laparoscopic abdominal aortic aneurysms repair. J Cardiovasc Surg (Torino). 2005;46:407-14.

6. Kolvenbach R, Ceshire N, Pinter L, Da Silva L, Deling O, Kasper AS. Laparoscopy-assisted aneurysm resection as a minimal invasive alternative in patients unsuitable for endovascular surgery. J Vasc Surg. 2001;34:216-21.

7. Ahn SS, Ro KM. Laparoscopic aortobifemoral bypass. Ann Vasc Surg. 1998;12:625-8.

8. Kolvenbach R, Da Silva L, Deling O, Schwierz E. Videoassisted aortic surgery. J Am Coll Surg. 2000;190:451-7.

9. Javerliat I, Coggia M, Di Centa I, Kitzis M, Mercier O, Goeau-Brissonniere O. Total laparoscopic abdominal aortic aneurysm repair with reimplantation of the inferior mesenteric artery. J Vasc Surg. 2004;39:1115-7.

10. Dion YM, Cardon A, Hartung O, Gracia CR. Laparoscopic Aorto-Iliac Surgery: Present Status and Future Perspectives. Surg Technol Int. 2000;VIII:201-207.

11. Ferrari M, Adami D, Del Corso A, et al. Laparoscopyassisted abdominal aortic aneurysm repair: early and middleterm results of a consecutive series of 122 cases. J Vasc Surg. 2006;43:695-700.

12. Mercier O, Coggia M, Javerliat I, Di Centa I, Colacchio G, Goeau-Brissonniere O. Total laparoscopic repeat aortic surgery. J Vasc Surg. 2004;40:822-5.

13. Kolvenbach R. The role of video-assisted vascular surgery. Eur J Vasc Endovasc Surg. 1998;15:377-9.

14. Silva L, Kolvenbach R, Pinter L. The feasibility of handassisted laparoscopic aortic bypass using a low transverse incision. Surg Endosc. 2002;16:173-6.
15. Wassiljew S, Kolvenbach R, Puerschel A, Schwierz E. Total laparoscopic iliac artery aneurysm repair using endoscopic techniques and endovascular balloon occlusion. Eur J Vasc Endovasc Surg. 2006;32:270-2.

16. Dion YM. History and perspectives in laparoscopic vascular surgery. Acta Chir Belg. 2004;104:493-8.

17. Munro MG. Laparoscopic access: complications, technologies, and techniques. Curr Opin Obstet Gynecol. 2002;14:36574.

18. Kolvenbach R, Deling O, Schwierz E, Landers B. Reducing the operative trauma in aortoiliac reconstructions--a prospective study to evaluate the role of video-assisted vascular surgery. Eur J Vasc Endovasc Surg. 1998;15:483-8.

19. Alfonsi P, Vieillard-Baron A, Coggia M, et al. Cardiac function during intraperitoneal $\mathrm{CO}_{2}$ insufflation for aortic surgery: a transesophageal echocardiographic study. Anesth Analg. 2006;102:1304-10.

20. Byrne J, Hallett JW Jr., Ilstrup DM. Physiologic responses to laparoscopic aortofemoral bypass grafting in an animal model. Ann Surg. 2000;231:512-8.

21. Polat C, Yilmaz S, Serteser M, Koken T, Kahraman A, Dilek ON. The effect of different intraabdominal pressures on lipid peroxidation and protein oxidation status during laparoscopic cholecystectomy. Surg Endosc. 2003;17:1719-22.

22. Coggia M, Javerliat I, Di Centa I, et al. Total laparoscopic versus conventional abdominal aortic aneurysm repair: a casecontrol study. J Vasc Surg. 2005;42:906-10; discussion 911.

23. Dion YM, Hartung O, Gracia C, Doillon C. Experimental laparoscopic aortobifemoral bypass with end-to-side aortic anastomosis. Surg Laparosc Endosc. 1999;9:35-8.

24. Fusco PEB, Marino HLT, Natal SRB, et al. Enxerto aortofemoral por via laparoscópica - modelo experimental. J Vasc Bras. 2005;4:396-400.

25. Dion YM, Gracia C. Experimental laparoscopic aortic aneurysm resection and aortobifemoral bypass. Surg Laparosc Endosc. 1996;6:184-90.

26. Dion YM, Gaillard F, Demalsy JC, Gracia CR. Experimental laparoscopic aortobifemoral bypass for occlusive aortoiliac disease. Can J Surg. 1996;39:451-5.

27. Dion YM, Chin AK, Thompson TA. Experimental laparoscopic aortobifemoral bypass. Surg Endosc. 1995;9:894-7.

28. Dion YM, Cardon A, Gracia CR, Doillon C. A model for laparoscopic aortic aneurysm resection. Surg Endosc. 1999; 13:654-7.

29. Dion YM, Thaveau F, Fearn SJ. Current modifications to totally laparoscopic "apron technique". J Vasc Surg. 2003;38:403-6.

30. Coggia M, Bourriez A, Javerliat I, Goeau-Brissonniere O. Totally laparoscopic aortobifemoral bypass: a new and simplified approach. Eur J Vasc Endovasc Surg. 2002;24:274-5.

31. Dion YM, Katkhouda N, Rouleau C, Aucoin A. Laparoscopy-assisted aortobifemoral bypass. Surg Laparosc Endosc. 1993;3:425-9.

32. Dion YM, Griselli F, Douville Y, Langis P. Early and midterm results of totally laparoscopic surgery for aortoiliac disease: lessons learned. Surg Laparosc Endosc Percutan Tech. 2004; 14:328-34. 
33. Kolvenbach R, Schwierz E, Wasilljew S, Miloud A, Puerschel A, Pinter L. Total laparoscopically and robotically assisted aortic aneurysm surgery: a critical evaluation. J Vasc Surg. 2004; 39:771-6.

34. Memon MA, Fitzgibbons RJ, Jr. Hand-assisted laparoscopic surgery (HALS): a useful technique for complex laparoscopic abdominal procedures. J Laparoendosc Adv Surg Tech A. 1998;8:143-50.

35. Zucker KA. Perceived future of laparoscopic general surgery. Can J Surg. 1992;35:297-304.

36. Kolvenbach R, Schwierz E. Combined endovascular/ laparoscopic approach to aortic pseudoaneurysm repair. J Endovasc Surg. 1998;5:191-3.

37. White GH, Yu W, May J, Chaufour X, Stephen MS. Endoleak as a complication of endoluminal grafting of abdominal aortic aneurysms: classification, incidence, diagnosis, and management. J Endovasc Surg. 1997;4:152-68.
38. Kolvenbach R, Lin J. Combining laparoscopic and endovascular techniques to improve the outcome of aortic endografts. Hybrid techniques. J Cardiovasc Surg (Torino). 2005;46:415-23.

Correspondência:

Ricardo de Alvarenga Yoshida

Departamento de Cirurgia e Ortopedia

Faculdade de Medicina de Botucatu - UNESP

CEP 18618-970 - Botucatu, SP

Tel.: (14) 3811.6269

E-mail: ricardoyoshida@gmail.com 